\title{
Computational Prediction of Diastereomeric Separation Behavior of Fluorescent $o$-Phthalaldehyde Derivatives of Amino Acids
}

\author{
Kenichiro Todoroki,* Tatsuki NaKano,* Hiroki Watanabe,* Jun Zhe Min,* Koichi InOue,* \\ Yoshinobu ISHIKAWA, ${ }^{* *}$ and Toshimasa TOYO’OKA*† \\ *Laboratory of Analytical and Bio-Analytical Chemistry, School of Pharmaceutical Sciences, \\ University of Shizuoka, 52-1 Yada, Suruga, Shizuoka 422-8526, Japan \\ **Laboratory of Physical Chemistry, School of Pharmaceutical Sciences, University of Shizuoka, 52-1 Yada, \\ Suruga, Shizuoka 422-8526, Japan
}

\begin{abstract}
We have developed a convenient method for predicting the LC resolution of amino acid diastereomers through computational calculations. For acquiring experimental data, we derivatized 10 amino acids using $o$-phthalaldehyde and $\mathrm{N}$-acetyl-L-cysteine as fluorogenic diastereomer-forming reagents and analyzed the diastereomers using reversed-phase LC and fluorescence detection. For theoretical chemical calculations, we used the publicly available semi-empirical calculation software MOPAC2012. Using the obtained experimental and theoretical data, we determined the change in analytical resolution with differences in the structure of the diastereomers. From the results obtained, we concluded that greater conformational change through diastereomeric derivatization induced an increase in the contact area with the stationary phase, leading to higher resolution.
\end{abstract}

Keywords Amino acid, chiral derivatization, computational calculation, diastereomer, liquid chromatography

(Received April 17, 2014; Accepted July 4, 2014; Published September 10, 2014)

\section{Introduction}

Most food ingredients, biological components, and pharmaceuticals are chiral compounds, and many of them exhibit differences in physiological function between enantiomers in vivo. Therefore, chiral analysis is invaluable in fields such as biological research, clinical diagnosis, and pharmaceutical development.

Among liquid chromatography (LC) separation methods for chiral compounds, precolumn derivatization methods to form diastereomers are practical and effective approaches and have been widely used in biomedical and food analyses. ${ }^{1}$ Although diastereomers generally differ in their physical and chemical properties, separating them using LC is quite difficult and the resolution varies depending on the analyte. In addition, it is very challenging to discriminate diastereomers with chiral centers separated by more than four bonds.,3 Therefore, a convenient predictive method using computational calculations to estimate the LC separation of diastereomers would be desirable.

Several previous studies have conducted molecular calculations simulating reversed-phase LC (RPLC) separation behavior. ${ }^{4-7}$ For instance, Hanai et al. calculated molecular interactions between analytes and modeled stationary phase compounds using molecular mechanics to quantitatively investigate basic phenomena in RPLC. ${ }^{5-7}$

Computational calculation studies of enantioseparation have

$\dagger$ To whom correspondence should be addressed.

E-mail: toyooka@u-shizuoka-ken.ac.jp also been reported. ${ }^{8-12}$ Several of these studies aimed to understand the chiral recognition mechanisms of chiral stationary phases such as $\beta$-cyclodextrin derivatives, ${ }^{10} \mathrm{~L}$-proline derivatives, ${ }^{11}$ and cinchona alkaloids. ${ }^{12}$

Recently, a great deal of research on chiral separation using molecular dynamics (MD) simulations ${ }^{13-16}$ and quantitative structure-enantioselective retention relationship calculations ${ }^{17,18}$ have been reported. For instance, Tsai et al. reported a prediction of in silico retention of peptide diastereomers using MD simulation. ${ }^{13,14}$ MD simulations to elucidate separation mechanism of chiral stationary phases such as polysaccharide ${ }^{15}$ and $N$-(3,5-dinitrobenzoyl)-S-leucine ${ }^{16}$ have been under intense investigation. Although MD simulation using thermodynamic parameters was capable of estimating interactions between the analytes and stationary phase compound, such an approach generally requires specialized software, high-spec computers, and considerable computational time.

Most of the above methods estimated the differences in the interactions between the analytes and the stationary phase to predict the separation behavior. In this study, we aimed to determine the mechanism by which fluorescent diastereomers that are generated using a fluorescence chiral derivatizing reagent are enantioseparated in RPLC. We hypothesized that it is possible to estimate the magnitude of chiral resolution solely from differences in the physical properties of the diastereomers in a separation system that does not involve electrostatic $\pi-\pi$ interactions between the analyte and the stationary phase. For the acquisition of the experimental data, we derivatized 10 amino acids using $o$-phthalaldehyde (OPA) and $\mathrm{N}$-acetyl-Lcysteine (NAC) as fluorogenic diastereomer-forming reagents ${ }^{19}$ (Fig. 1) and analyzed the derivatives by RPLC and fluorescence 


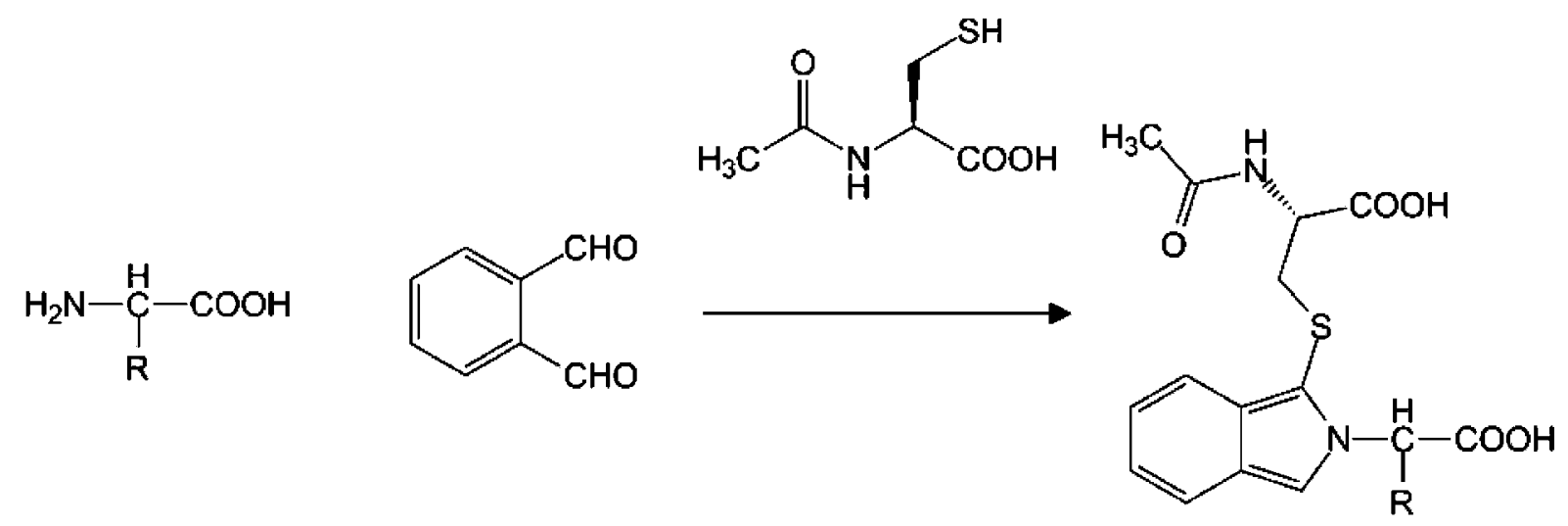

Fig. 1 Fluorescence diastereomeric derivatization of amino acids using $o$-phthalaldehyde and $N$-acetyl-L-cysteine.

detection. For the theoretical chemical calculations, we used the publicly available semi-empirical calculation software, MOPAC2012.20,21 From the obtained experimental and theoretical data, we evaluated the dependence of the resolution on the structure of the diastereomers. Using octadecane as a pseudo-stationary phase compound in an octadecyl silica column, we also investigated the change in resolution due to the differences in the structures of diastereomer-octadecane complexes. To the best of our knowledge, this is the first report describing the computational prediction of the diastereomeric separation behavior of fluorescent OPA derivatives of amino acids.

\section{Experimental}

\section{Reagents and chemicals}

Deionized and distilled water, purified using an Aquarius PWU-200 automatic water distillation apparatus (Advantec, Tokyo, Japan), was used to prepare all aqueous solutions. OPA and NAC were purchased from Wako (Osaka, Japan). Stock solutions of D- and L-amino acids $(5 \mathrm{mM})$ were prepared in water. All stock solutions were stored at $4{ }^{\circ} \mathrm{C}$ and then diluted further with water to the desired concentration prior to use. All other chemicals were of the highest purity available and were used as received.

\section{HPLC system}

A Nexera ultra high performance liquid chromatograph (UPLC) system (Shimadzu Corp., Kyoto, Japan) was used for the analyses, consisting of a CBM-20A system controller, an SIL-30AC auto sampler, two LC-30AD pumps, a DGU-20A online degasser, a CTO-30A column oven, and an RF-20A fluorescence spectrometer, equipped with a $12-\mu \mathrm{L}$ flow cell. Fluorescence intensity was monitored at excitation and emission wavelengths of 350 and $450 \mathrm{~nm}$, respectively. The collected data were processed by Lab Solutions LC Ver. 1.21 (Shimadzu) and peak areas and heights were quantified using the baselineto-baseline method. The RPLC column used was TSK gel ODS-100V $(3 \mu \mathrm{m}, 150 \times 2.0 \mathrm{~mm}$; Tosoh, Tokyo, Japan $)$. The mobile phases used were aqueous acetonitrile containing $0.1 \%$ formic acid with acetonitrile contents of $10 \%$ (Ser), $15 \%$ (Thr), $20 \%$ (Ala and Tyr), 25\% (Met), 30\% (Val and Trp), and 35\% (Phe, Ile, and Leu). The flow rate of the mobile phases was $0.2 \mathrm{~mL} / \mathrm{min}$ and the column oven temperature was $40^{\circ} \mathrm{C}$.

\section{Fluorogenic OPA chiral derivatization procedure}

A $10-\mu \mathrm{L}$ aliquot of the amino acid solution, $100 \mathrm{mM}$ borate

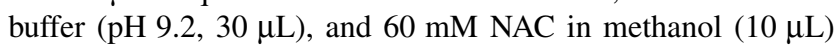
were added to an empty vial in that order. The solution was well mixed and held at room temperature for $2 \mathrm{~min}$.

\section{Computational calculations}

The structures of the amino acid derivatives, the octadecane and these complexes were constructed using Chem3D Pro v. 12.0 (CambridgeSoft, Cambridge, MA) and energetically optimized using the molecular mechanics method and quantum chemical calculations. The semi-empirical calculations were carried out by the MOPAC2012 program. Geometric calculations under vacuum were carried out using the PM7 method (Stewart, 2013). All geometries were completely optimized (keyword PRECISE, equivalent to GNORM $=0.01$ and MMOK) by the eigenvector-following routine (keyword $\mathrm{EF}$ ). The dielectric constants (keyword EPS) for the solvents were set to 74.1 ( $10 \%$ acetonitrile), 72.0 (15\% acetonitrile), 69.8 (20\% acetonitrile), 67.7 (25\% acetonitrile), 65.6 (30\% acetonitrile), and 63.5 (35\% acetonitrile). For elucidating optimized geometries of complexes of the amino acid derivatives and the pseudo-stationary phase compound octadecane, we started each calculation from an initial structure. In the structure, two atomic distance between carbon atom connecting to sulfur atom in the fluorescent isoindole structure and carbon atom at 9 -position of octadecane was set at $3 \times 10^{-10} \mathrm{~m}$. Based on the optimized geometries, heat of formation (HOF) energies $(\mathrm{kJ} / \mathrm{mol})$, molecular surface areas $\left(\mathrm{m}^{2}\right)$, and dipole moments (Debye) were calculated. Winmostar Version 4.02 (http:// winmostar.com/) was used to visualize and compare the optimized structures.

\section{Results and Discussion}

\section{Diastereomeric LC separation of racemic amino acids}

Table 1 summarizes the retention times $\left(t_{\mathrm{R}}\right)$, retention factor $(k)$, resolution of the diastereomers $\left(R_{\mathrm{s}}\right)$, separation coefficient $(\alpha)$, and initial eluate for the examined compounds. Among the amino acids, Val, Met, Trp, Phe, Ile, and Leu exhibited particularly large resolutions $\left(R_{\mathrm{s}}>1.5\right)$ under these conditions. In contrast, the hydrophilic amino acids (Ser, Ala, Thr, and Tyr) could not be fully enantioseparated. The first eluates of all of the derivatives were their L-forms, except for Thr. 
Dependence of enantioseparation on the structures of diastereomers

The structures of the fluorescent diastereomer derivatives were energetically optimized using the molecular mechanics method and quantum chemical calculations. The semi-empirical calculations were carried out by the MOPAC2012 program. Table 2 shows the calculated data for the structural properties of the amino acid derivatives in the same polar solvents as mobile phases.

Figure 2 shows the optimized structures for the Ile, Ala, and Tyr derivatives. Diastereomeric derivatization with OPA and NAC, the structures of the D- and L-Ile derivatives near their isobutyl groups. The HOFs of the derivatives were -918.038

Table 1 Experimental data for LC separation of amino acid derivatives

\begin{tabular}{lrrrrrrrr}
\hline $\begin{array}{c}\text { Amino } \\
\text { acid }\end{array}$ & $\begin{array}{c}t_{\mathrm{R} 1} \\
\mathrm{~min}\end{array}$ & $\begin{array}{c}t_{\mathrm{R} 2} \\
\mathrm{~min}\end{array}$ & $k_{1}$ & $k_{2}$ & $\alpha$ & $R_{\mathrm{s}}$ & $\begin{array}{c}\text { Initial } \\
\text { eluate }\end{array}$ & $\begin{array}{c}\mathrm{MeCN} \\
\text { content, } \\
\%\end{array}$ \\
\hline Ser & 40.51 & 42.83 & 18.14 & 19.24 & 1.06 & 1.06 & $\mathrm{~L}$ & 10 \\
$\mathrm{Thr}$ & 32.76 & 34.49 & 14.85 & 15.68 & 1.06 & 1.26 & $\mathrm{D}$ & 15 \\
Ala & 29.53 & 31.02 & 13.57 & 14.31 & 1.05 & 0.87 & $\mathrm{~L}$ & 20 \\
Tyr & 51.14 & 53.26 & 24.13 & 25.17 & 1.04 & 1.11 & $\mathrm{~L}$ & 20 \\
Met & 36.41 & 39.52 & 17.42 & 19.00 & 1.09 & 2.28 & $\mathrm{~L}$ & 25 \\
Val & 20.02 & 22.48 & 9.06 & 10.30 & 1.14 & 3.54 & $\mathrm{~L}$ & 30 \\
Trp & 24.27 & 26.93 & 11.60 & 12.99 & 1.12 & 2.97 & $\mathrm{~L}$ & 30 \\
Phe & 27.89 & 29.55 & 18.13 & 19.27 & 1.06 & 1.67 & $\mathrm{~L}$ & 30 \\
Ile & 32.29 & 37.15 & 15.77 & 18.29 & 1.16 & 4.11 & $\mathrm{~L}$ & 30 \\
Leu & 37.10 & 39.50 & 18.26 & 19.51 & 1.07 & 1.85 & $\mathrm{~L}$ & 30 \\
\hline
\end{tabular}

(A) $-918.038 \mathrm{~kJ} / \mathrm{mol}$
$4.21743 \times 10^{-22} \mathrm{~m}^{2}$

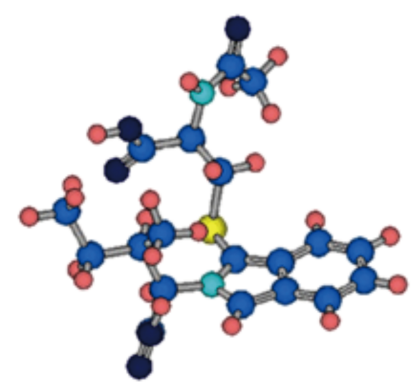

D-lle

$$
\begin{gathered}
-938.630 \mathrm{~kJ} / \mathrm{mol} \\
4.22801 \times 10^{-22} \mathrm{~m}^{2}
\end{gathered}
$$

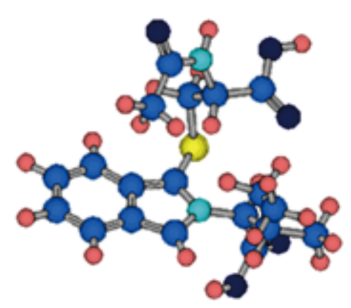

L-Ile
(D-form) and $-938.630 \mathrm{~kJ} / \mathrm{mol}$ ( $\mathrm{L}-$ form), a difference of $20.592 \mathrm{~kJ} / \mathrm{mol}$. In contrast, the HOFs of the D- and L-Ala derivatives were -866.592 (D-form) and $-865.805 \mathrm{~kJ} / \mathrm{mol}$ (L-form), a difference of $0.787 \mathrm{~kJ} / \mathrm{mol}$. Figure 3 shows the

Table 2 Calculated structural properties of amino acid derivatives

\begin{tabular}{lccccc}
\hline Derivative & $\begin{array}{c}\text { Dipole } \\
\text { moment/ } \\
\text { debye }\end{array}$ & $\begin{array}{c}\mathrm{HOF} / \\
\mathrm{kJ} \mathrm{mol}^{-1}\end{array}$ & $\begin{array}{c}\Delta \mathrm{HOF} / \mathrm{kJ} \mathrm{mol}^{-1} \\
\text { \% }\end{array}$ & $\begin{array}{c}\mathrm{SCR}, \\
\text { Molecular } \\
\left(\times 10^{-22} \mathrm{~m}^{2}\right)\end{array}$ \\
\hline D-Ser & 4.879 & -1056.450 & 8.544 & 0.809 & 3.72150 \\
L-Ser & 9.382 & -1064.994 & & & 3.69805 \\
D-Thr & 9.164 & -1080.951 & 2.989 & 0.276 & 3.99725 \\
L-Thr & 5.153 & -1077.962 & & & 3.87434 \\
D-Ala & 6.918 & -866.592 & 0.787 & 0.091 & 3.66228 \\
L-Ala & 9.274 & -865.805 & & & 3.65862 \\
D-Tyr & 9.258 & -956.219 & 19.750 & 2.065 & 4.55439 \\
L-Tyr & 6.088 & -975.969 & & & 4.5417 \\
D-Met & 5.582 & -863.653 & 5.241 & 0.607 & 4.29061 \\
L-Met & 3.440 & -868.894 & & & 4.27594 \\
D-Val & 5.059 & -899.934 & 19.314 & 2.146 & 4.03601 \\
L-Val & 3.716 & -919.248 & & & 4.0195 \\
D-Trp & 6.691 & -732.019 & 14.040 & 1.918 & 4.68739 \\
L-Trp & 8.199 & -717.979 & & & 4.60988 \\
D-Phe & 6.926 & -749.881 & 9.795 & 1.306 & 4.43886 \\
L-Phe & 4.703 & -759.676 & & & 4.47068 \\
D-Ile & 5.113 & -918.038 & 20.592 & 2.243 & 4.21743 \\
L-Ile & 4.294 & -938.630 & & & 4.22801 \\
D-Leu & 3.441 & -935.992 & 3.675 & 0.393 & 4.28446 \\
L-Leu & 4.780 & -932.317 & & & 4.28675 \\
\hline
\end{tabular}

$-865.805 \mathrm{~kJ} / \mathrm{mol}$ $3.66228 \times 10^{-22} \mathrm{~m}^{2}$ $3.65862 \times 10^{-22} \mathrm{~m}^{2}$

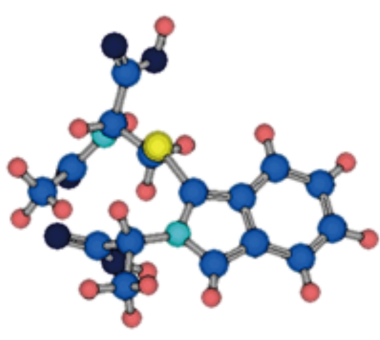

D-Ala

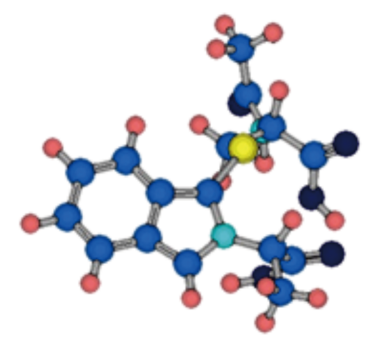

L-Ala

(C)

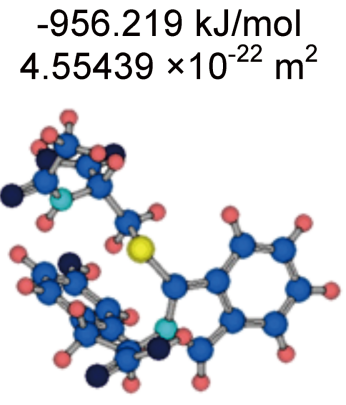

D-Tyr
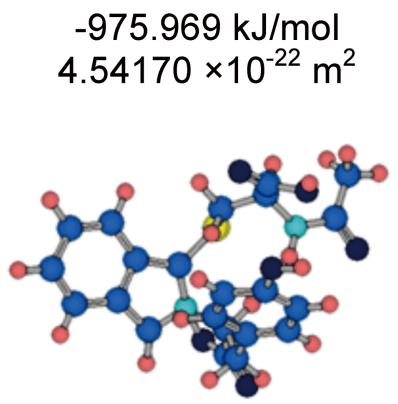

L-Tyr

Fig. 2 Optimized 3D structures, $\mathrm{HOF}(\mathrm{kJ} / \mathrm{mol})$ and molecular surface area $\left(\mathrm{m}^{2}\right)$ of Ile (A), Ala (B), and $\operatorname{Tyr}(\mathrm{C})$ derivatives. 
(A)

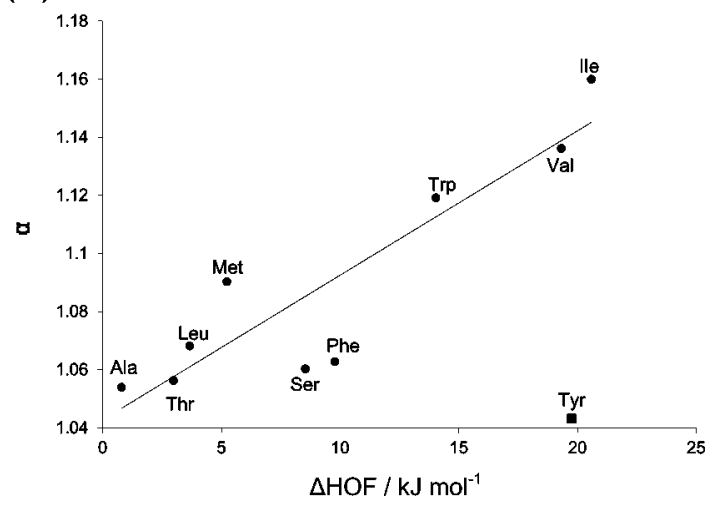

(C)

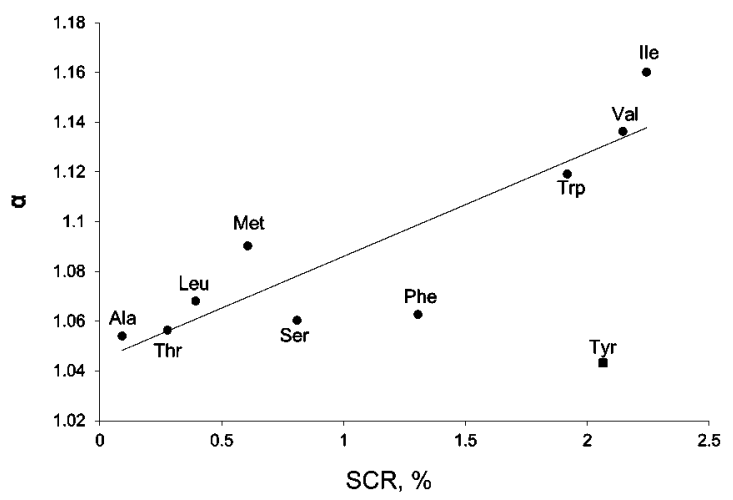

(B)

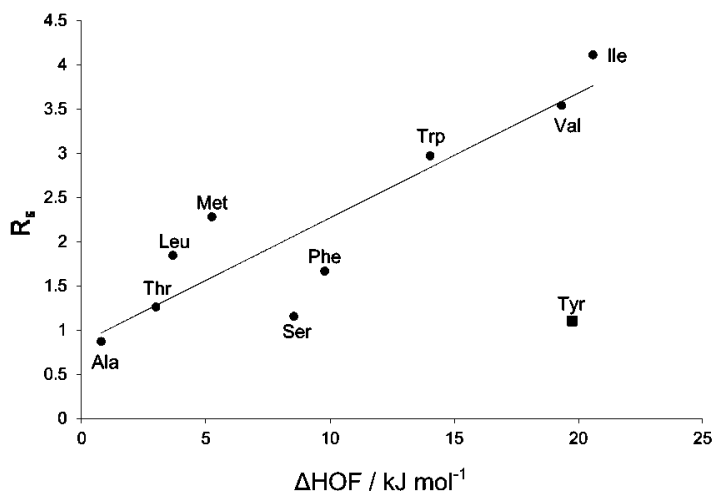

(D)

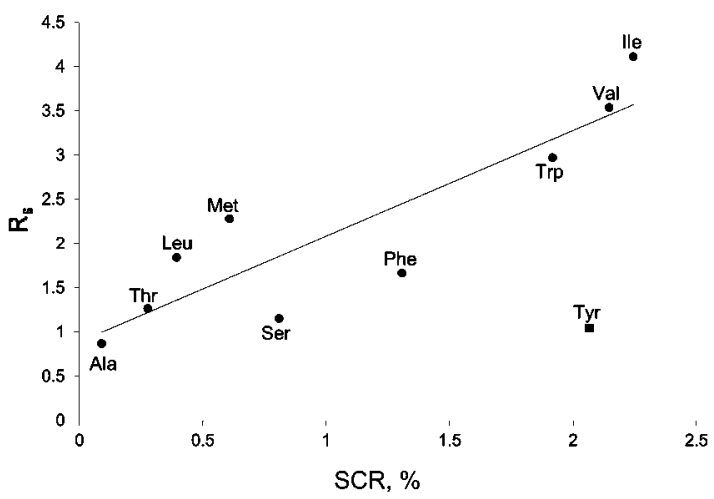

Fig. 3 Differences in the HOFs of D- and L-forms versus $\alpha(\mathrm{A})$ and $R_{\mathrm{S}}(\mathrm{B})$. Differences in the SCRs of D- and L-forms versus $\alpha(\mathrm{C})$ and $R_{\mathrm{S}}(\mathrm{D})$. Regression equations and correlation coefficients: (A) $y=$ $0.005 x+1.04218, r=0.9038$; (B) $y=0.1412 x+0.8564, r=0.8971$; (C) $y=0.0416 x+1.0444, r=$ 0.8870 ; (D) $y=1.1946 x+0.8904, r=0.8883$.

Table 3 Calculated structural properties of complexes of amino acid derivatives and octadecane

\begin{tabular}{|c|c|c|c|c|c|c|c|}
\hline Derivative & $\begin{array}{l}\text { HOF of } \\
\text { complex/ } \\
\mathrm{kJ} \mathrm{mol}^{-1}\end{array}$ & $\begin{array}{c}\Delta \mathrm{HOF} \text { of } \\
\text { complex/ } \\
\mathrm{kJ} \mathrm{mol}^{-1}\end{array}$ & $\begin{array}{c}\mathrm{SCR}, \\
\%\end{array}$ & $\begin{array}{l}\Sigma \text { Molecular } \\
\text { surface area } \\
\left(\times 10^{-22} \mathrm{~m}^{2}\right)\end{array}$ & $\begin{array}{l}\text { Molecular } \\
\text { surface area } \\
\left(\times 10^{-22} \mathrm{~m}^{2}\right)\end{array}$ & $\begin{array}{c}\text { VCA } \\
\left(\times 10^{-24} \mathrm{~m}^{2}\right)\end{array}$ & $\begin{array}{c}\Delta \mathrm{VCA} \\
\left(\times 10^{-24} \mathrm{~m}^{2}\right)\end{array}$ \\
\hline D-Ser & -1518.255 & \multirow{2}{*}{17.322} & \multirow{2}{*}{1.141} & 7.92575 & 7.78429 & 14.146 & \multirow{2}{*}{8.690} \\
\hline L-Ser & -1500.933 & & & 7.90230 & 7.84774 & 5.456 & \\
\hline D-Thr & -1547.122 & \multirow{2}{*}{12.554} & \multirow{2}{*}{0.811} & 8.20150 & 8.03321 & 16.829 & \multirow{2}{*}{6.561} \\
\hline L-Thr & -1534.568 & & & 8.07859 & 7.84469 & 23.390 & \\
\hline D-Ala & -1340.302 & \multirow{2}{*}{5.262} & \multirow{2}{*}{0.392} & 7.86653 & 7.46101 & 40.552 & \multirow{2}{*}{7.429} \\
\hline L-Ala & -1345.564 & & & 7.86287 & 7.53164 & 33.123 & \\
\hline D-Tyr & -1408.095 & \multirow{2}{*}{26.942} & \multirow{2}{*}{1.914} & 8.75864 & 8.51555 & 24.309 & \multirow{2}{*}{4.165} \\
\hline L-Tyr & -1435.037 & & & 8.74595 & 8.54451 & 20.144 & \\
\hline D-Met & -1344.743 & \multirow{2}{*}{19.692} & \multirow{2}{*}{1.765} & 8.49486 & 8.19002 & 30.484 & \multirow{2}{*}{8.821} \\
\hline L-Met & -1364.435 & & & 8.48019 & 8.26356 & 21.663 & \\
\hline D-Val & -1385.168 & \multirow{2}{*}{24.451} & \multirow{2}{*}{0.392} & 8.24026 & 7.98519 & 25.507 & \multirow{2}{*}{12.797} \\
\hline L-Val & -1409.619 & & & 8.22375 & 8.09665 & 12.710 & \\
\hline D-Trp & -1198.274 & \multirow{2}{*}{6.295} & \multirow{2}{*}{1.464} & 8.89164 & 8.60159 & 29.005 & \multirow{2}{*}{10.436} \\
\hline L-Trp & -1204.569 & & & 8.81413 & 8.62844 & 18.569 & \\
\hline D-Phe & -1223.457 & \multirow{2}{*}{9.762} & \multirow{2}{*}{0.525} & 8.64311 & 8.35663 & 28.648 & \multirow{2}{*}{5.499} \\
\hline L-Phe & -1233.219 & & & 8.67493 & 8.44344 & 23.149 & \\
\hline D-Ile & -1404.884 & \multirow{2}{*}{2.683} & \multirow{2}{*}{0.191} & 8.42168 & 8.17227 & 24.941 & \multirow{2}{*}{11.809} \\
\hline L-Ile & -1402.201 & & & 8.43226 & 8.30094 & 13.132 & \\
\hline D-Leu & -1414.663 & \multirow{2}{*}{27.657} & \multirow{2}{*}{1.955} & 8.48871 & 8.23735 & 25.136 & \multirow{2}{*}{9.509} \\
\hline L-Leu & -1442.320 & & & 8.49100 & 8.33473 & 15.627 & \\
\hline
\end{tabular}

a. Surface area of octadecane was calculated as $4.20425 \times 10^{-22} \mathrm{~m}^{2}$. 
(A)

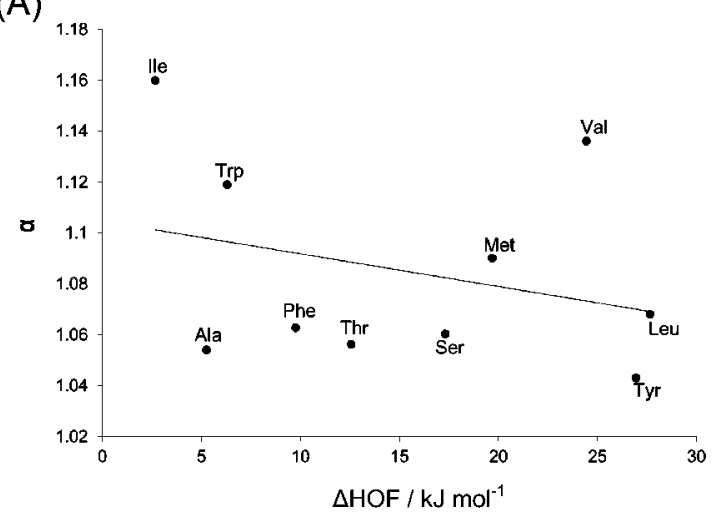

(C)

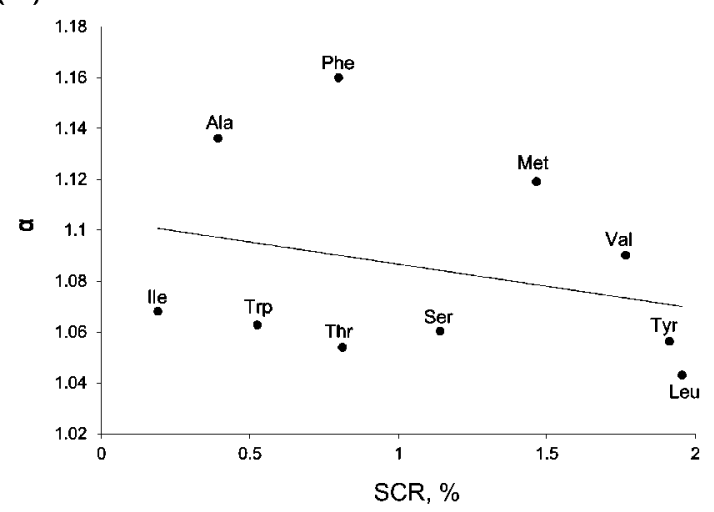

(B)

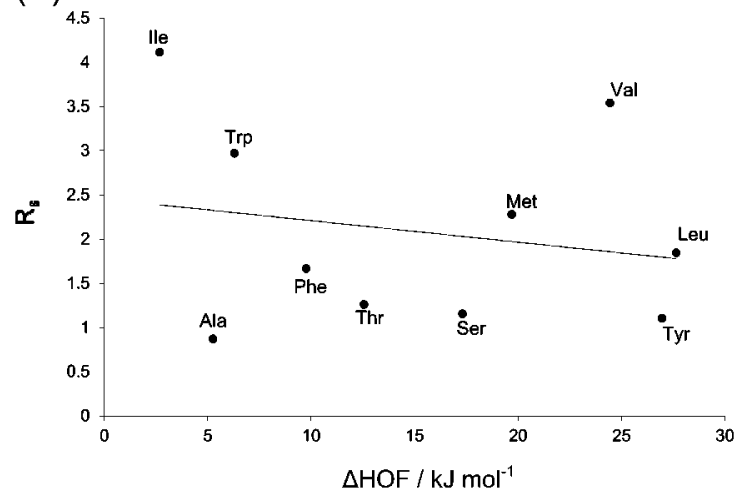

(D)

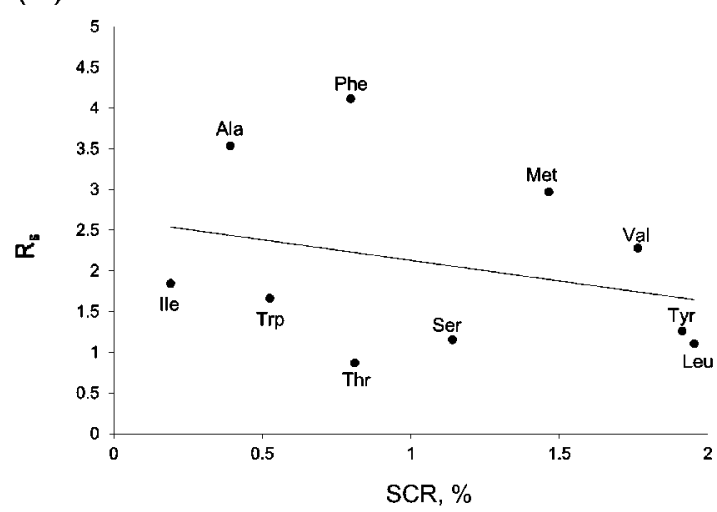

Fig. 4 Differences in the HOFs of octadecane complexes of D- and L-forms versus $\alpha(\mathrm{A})$ and $R_{\mathrm{s}}(\mathrm{B})$. Differences in the SCRs of octadecane complexes of D- and L-forms versus $\alpha(\mathrm{C})$ and $R_{\mathrm{S}}(\mathrm{D})$. Regression equations and correlation coefficients: (A) $y=-0.0013 x+1.1046, r=0.2980$; (B) $y=-0.0244 x+$ $2.4539, r=0.2027$; (C) $y=-0.0174 x+1.1041, r=0.2825$; (D) $y=-0.56056 x+2.6352, r=0.2939$.

difference in the HOFs of the D- and L-forms versus the $\alpha$ and $R_{\mathrm{S}}$ values. Diastereomers with large differences in the HOFs had high $\alpha$ and $R_{\mathrm{S}}$ values (other than Tyr), with strong correlations ( $r=0.9038$ and 0.897$)$.

To represent these differences, we defined the structural change rate (SCR) using the following equation:

$$
\begin{array}{r}
\operatorname{SCR}(\%)=\left(\left|\mathrm{HOF}_{\mathrm{D} \text {-form derivative }}-\mathrm{HOF}_{\mathrm{L} \text {-form derivative }}\right| /\right. \\
\left.\left|\mathrm{HOF}_{\mathrm{D} \text {-form derivative }}\right|\right) \times 100
\end{array}
$$

The SCR versus $\alpha$ and $R_{\mathrm{S}}$ are plotted in Figs. 3C and 3D; these factors were strongly correlated ( $r=0.8870$ and 0.8883 ), except for Tyr, consistent with the HOFs. The SCR of the Ala derivative was $0.09 \%$, while that of the Ile derivative was $2.84 \%$; they differed by 26.2 times due to differences in the side chain structures of the amino acids.

Because diastereomers are the same atomic and electron numbers, the difference of HOF energies are thought to be a parameter representing the differences of conformation. The difference of conformation between the diastereomers should lead to differences in dipole moments and interactions with the ODS stationary phase. These results suggest that the magnitude of the difference in conformation between the enantiomers strongly affects their resolution in the chromatograms.

Dependence of enantioseparation on the structures diastereomers and pseudo-stationary phase compound

Table 3 summarizes the calculated structural properties for complexes of the amino acid derivatives and the pseudostationary phase compound octadecane. Figure 4 shows the differences in the HOFs of the octadecane complexes of the D- and L-forms versus $\sigma$ and $R_{\mathrm{s}}$. Unlike the results for the enantiomers alone, poor correlations $(r=0.298$ and 0.203$)$ were obtained. When calculating the octadecane complexes, the HOF energy of octadecane contributed greater than the difference of HOF energy between diastereomers in total energy; this may resulted in the poor correlations.

The virtual contact area (VCA) was calculated by subtracting the molecular surface area of the complex from the sum of those of the amino acid derivatives and octadecane. Because there were different $\mathrm{LC}$ conditions for each amino acid derivative, we could not determine a correlation between the retention time and the molecular surface area of the derivatives.

On the other hand, the differences in the VCAs of the octadecane complexes of the D- and L-forms versus $\alpha$ and $R_{\mathrm{s}}$ showed good correlations ( $r=0.867$ and 0.827) (Fig. 5). In addition, of the two diastereomer complexes, that with the larger VCA was eluted later for all derivatives examined.

Based on these results, greater conformational change by diastereomeric derivatization induced an increase in contact area with the stationary phase, resulting in higher resolution.

\section{Conclusions}

We have demonstrated that it is possible to conveniently predict 
(A)

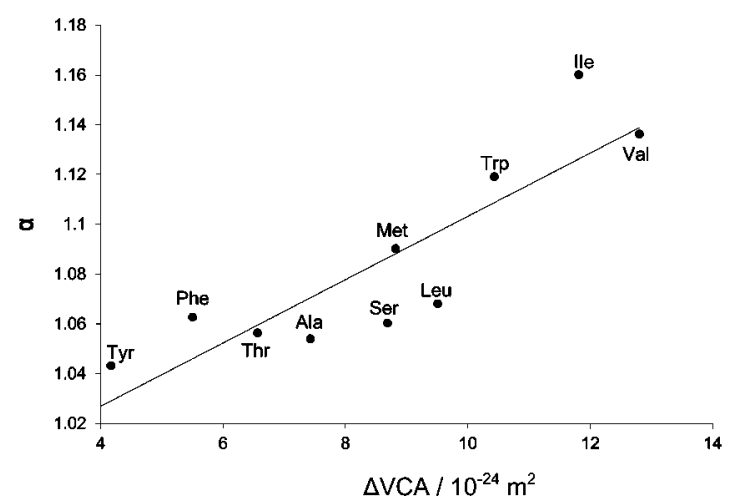

(B)

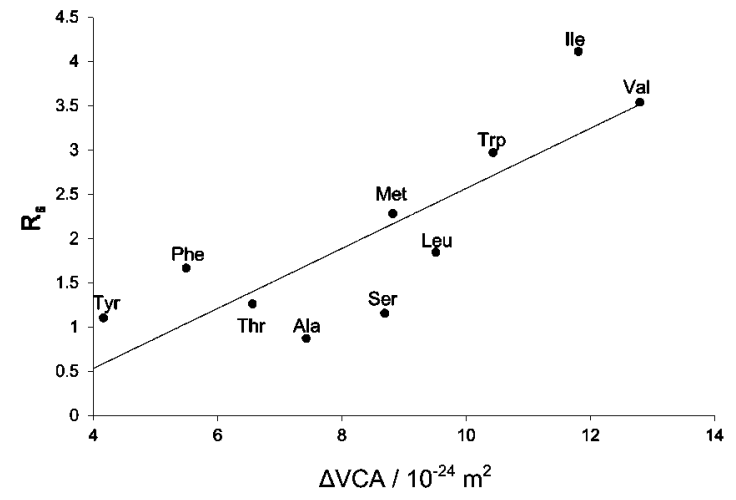

Fig. 5 Differences in the VCAs of octadecane complexes of D- and L-forms versus $\alpha(\mathrm{A})$ and $R_{\mathrm{s}}(\mathrm{B})$. Regression equations and correlation coefficients: (A) $y=0.0127 x+0.9761, r=0.8679$; (B) $y=$ $0.3391 x-0.8255, r=0.8274$.

the LC resolution of OPA amino acid diastereomers by a semiempirical molecular orbital method. This method does not require MD calculations or molecular simulations that take into account interactions with the stationary phase. The results showed that greater conformational change through diastereomeric derivatization induced an increase in contact area with the stationary phase, resulting in higher resolution.

Our method will be useful in various fields using chiral LC analysis, such as development of high-resolution chiral discriminating reagents and prediction of separation behavior of D-amino acid-containing peptides.

\section{Acknowledgements}

This study was supported in part by a Grant-in-Aid for Scientific Research (C) (25460040) from the Ministry of Education, Culture, Sports, Science, and Technology of Japan.

\section{References}

1. T. Toyo'oka, "Modern Derivatization Methods for Separation Sciences", 1999, John Wiley and Sons, Chichester.

2. H. Ohrui, Bunseki Kagaku, 2004, 53, 805.

3. H. Ohrui, Anal. Sci., 2008, 24, 31.

4. R. K. Lindsey, J. L. Rafferty, B. L. Eggimann, J. I. Siepmann, and M. R. Schure, J. Chromatogr. A, 2013,
1287, 60.

5. T. Hanai, Anal. Bioanal. Chem., 2005, 382, 708.

6. T. Hanai and H. Homma, Anal. Bioanal. Chem., 2008, 390, 369.

7. T. Hanai, J. Chromatogr. Sci., 2014, 52, 75.

8. K. B. Lipkowitz, J. Chromatogr. A, 1995, 694, 15.

9. K. B. Lipkowitz, J. Chromatogr. A, 2001, 906, 417.

10. J. H. Shi, Z. J. Ding, and Y. Hu, Chromatographia, 2011, $74,319$.

11. Z. Chilmonczyk, H. Ksycińska, J. Cybulski, M. Rydzewski, and A. Leś, Chirality, 1998, 10, 821.

12. S. Keunchkarian, C. A. Franca, L. G. Gagliardi, and C. B. Castells, J. Chromatogr. A, 2013, 1298, 103.

13. C. W. Tsai, C. I. Liu, Y. C. Chan, H. H. G. Tsai, and R. C. Ruaan, J. Phys. Chem. B, 2010, 114, 11620.

14. C. W. Tsai, W. Y. Chen, and R. C. Ruaan, Langmuir, 2012, $28,13601$.

15. A. Zhang, W. Lai, J. Sun, G. Hu, and W. Liu, J. Chromatogr. A, 2013, 1281, 26.

16. B. Liu, Y. Zhou, G.-S. Yang, and H. Y. Aboul-Enein, Chirality, 2013, 25, 101.

17. M. Lämmerhofer, J. Chromatogr. A, 2010, 1217, 814.

18. B. Rasulev, M. Turabekova, M. Gorska, K. Kulig, A. Bielejewska, J. Lipkowski, and J. Leszczynski, Chirality, 2012, 24, 72.

19. N. Nimura and T. Kinoshita, J. Chromatogr. A, 1986, 352, 169.

20. J. J. P. Stewart, J. Comput.-Aided. Mol. Des., 1990, 4, 1.

21. J. J. P. Stewart, J. Mol. Model., 2013, 19, 1. 